\title{
Agôn
}

Revue des arts de la scène

7| 2015

La Distribution

\section{Processus de distribution dans/de la performance} post-pornographique

Entretien réalisé par Barbara Métais-Chastanier

Matthieu Hocquemiller et Barbara Métais-Chastanier

\section{(2) OpenEdition}

\section{Journals}

Édition électronique

URL : http://journals.openedition.org/agon/3262

DOI : 10.4000/agon.3262

ISSN : 1961-8581

Éditeur

Association Agôn

Référence électronique

Matthieu Hocquemiller et Barbara Métais-Chastanier, « Processus de distribution dans/de la performance post-pornographique », Agôn [En ligne], 7 | 2015, mis en ligne le 12 novembre 2015, consulté le 15 septembre 2020. URL : http://journals.openedition.org/agon/3262

Ce document a été généré automatiquement le 15 septembre 2020.

Association Agôn et les auteurs des articles 


\title{
Processus de distribution dans/de la performance post-pornographique
}

\author{
Entretien réalisé par Barbara Métais-Chastanier
}

Matthieu Hocquemiller et Barbara Métais-Chastanier

Chorégraphe et militant queer, Matthieu Hocquemiller interroge depuis plusieurs années le corps comme espace politique. Pour ce faire il questionne aussi les processus de distribution tant au niveau du rapport à l'interprète qu'au niveau des effets de légitimation ou d'exclusion des formes dominantes et des cultures marginalisées ou invisibilisées. Dans ses deux dernières créations, (nou) créée en 2014 au festival de Montpellier danse et Auto-Porn Box présentée à hTh en mai 2015, il s'interroge sur la sexualité et travaille à l'élaboration d'imaginaire sexuels alternatifs en s'appuyant sur les expériences et les discours de subjectivités radicales : performeur-se et militant-e queer, travailleur et travailleuse du sexe, danseur-e ou universitaire se mettent au service d'une écriture chorégraphique qui cherche à voir ce que l'intime a de politique et comment il s'élabore comme culture et comme pratique. Cette année, aux côtés de Marianne Chargois, il impulse la programmation du festival Explicit <http://festivalexplicit.fr/wpexplicit/> en mai à hTh, croisant culture et contre-culture autour du sexuel envisagé comme objet politique et social. Habité par le souci de troubler les identités, pour gagner par l'activation de possibilités représentatives d'autres espaces et de nouvelles formes de subjectivation, il revient dans cet entretien sur les différentes dimensions que prend la distribution dans sa pratique de chorégraphe, d'interprète et de programmateur.

\section{La distribution comme rencontre provoquée}

Barbara MÉTAIS-CHASTANIER. II me semblait bien de commencer cet entretien par une question simple : est-ce que tu pourrais me donner ta définition de la distribution, en sachant qu'elle a des enjeux aussi bien artistiques, qu'économiques ou politiques? II me semble que la distribution prend souvent dans tes pièces la forme de la rencontre, qu'en penses-tu?

Matthieu HOCQUEMILLER. Je crois que cela dépend des projets et de ce qui doit se dire et avec qui. Mais effectivement, il y a souvent chez moi la volonté de sortir des réseaux 
de danse formatés ou formatant et donc la distribution procède souvent de la rencontre, même s'il s'agit de rencontres provoquées. Quand je dis que ça dépend des projets, c'est parce que je cherche des corps qui soient traversés par certaines choses, qui soient porteurs d'une certaine histoire ou d'un certain discours. Pour les deux derniers projets par exemple, cela m'a paru important par rapport à l'endroit d'où l'on parle. Le travers de cette démarche, s'il n'est pas assumé comme tel, peut être la dimension documentaire. À l'inverse dans Auto-porn box (2015), il y a un côté documentaire très assumé. Là pour le coup c'est un point de départ : c'est un focus sur la sexualité par l'autoportrait, l'entrée étant choisie par le performeur. Par exemple Kay [Garnellen] qui a travaillé sur sa transition de genre a pris une piste documentaire : il y avait ses seringues de testostérone, ses photos de transition. Et moi je me suis mis au service de ça. Marianne [Chargois], elle, est dominatrice et c'était un travail documentaire sur une session avec ses clients. Donc là on est vraiment dans du document. Mais ça mis à part, je cherche plutôt à travailler avec des gens qui sont porteurs d'un point de vue ou d'une histoire parce que le processus de travail et les signes qui vont être émis à partir de là n'auront pas le même sens.

B. MC. Est-ce que tu dirais que l'enjeu c'était, avec (nou) où la distribution s'appuyait sur des travailleurs du sexe, des performeurs queer, etc., de chercher un point d'énonciation extérieur à la danse ou plutôt de travailler dans un champ social et culturel à défaire un imaginaire dominant, des stéréotypes du corps et des sexualités?

M. H. Il y a des danseurs dans la pièce, en plus des performeurs, et ce qui m'intéressait c'était moins la référence à la danse, que le fait que la façon de travailler, d'émettre des signes, allait être différente si la pièce était interprétée par des artistes ou par des personnes ayant traversé les problématiques de la sexualité et du genre, par des personnes qui ont aussi envie de le travailler sous l'angle de la représentation. Ça permet d'avoir une parole située et également de ne pas reproduire des stéréotypes, notamment à travers la présence de corps non-normés.

B. M.C Dans Jusque là c'est nous (2012), c'est ce qui m'avait frappée, cette confrontation avec des corps qu'on voit rarement sur les plateaux de danse et qui se charge d'un sens politique.

M.H. Si on prend au pied de la lettre l'idée que le corps du danseur est porteur des signes qu'il émet, il me semble que certains des corps, comme celui de Hurlande, dans Jusque là c'est nous, permettent d'émettre d'autres signes, sans être dans le documentaire. Et pas du tout sous un angle victimisant ou fragilisant.

B. MC. II me semble cependant que tu n'explicites pas dans tes pièces chorégraphiques ce qui t'a amené à provoquer ces rencontres?

M.H. Dans Jusque là c'est nous, en effet, je n'avais pas envie d'être dans une logique de témoignage, qui aurait raconté la situation de Hurlande, migrante et sans-papier. Ce n'était pas le propos.

B. MC. Mais c'est une chose que tu as retrouvée ensuite dans Auto-porn Box?

HM. Pour Auto-Porn Box, cela s'appuie sur un choix de distribution antérieur puisque cela remonte à (nou) : j'avais des exigences particulières pour cette pièce : j'avais envie que ce soit des gens politiquement très conscients. Alors de fait, ils sont soit militants ou militantes soit concernés par la question politique et ils sont tous très conscients des endroits de genre et de sexualité, conscients aussi de la manière dont ils mettent en scène leur propre corps. Donc ce n'est pas moi qui apportais les problématiques sexuelles ou l'urgence d'en parler. Kay [Garnellen] est trans, 
travailleur du sexe et militant pour ces deux causes; Marianne [Chargois] est militante et travailleuse du sexe; Mathieu [Jedrazac] est impliqué dans un militantisme queer et LGBT; Tom est gay et escort avec un mode de vie orienté politiquement; Camille [Mutel] est peut-être celle qui aurait le profil le moins politique mais dans son travail solo à elle il y a aussi du sexuellement explicite et elle s'interrogeait sur la mise en scène de sa sexualité. Et ça c'était aussi une condition pour la distribution de (Nou) : je n'avais jamais travaillé, à part sur moi-même, sur le sexuellement explicite et j'avais fait la première Auto Porn box en travaillant sur la masturbation, cette pratique renvoyée au non-représentable et à l'intime, et même à l'intime de l'intime puisque c'est un exercice solitaire. Qui plus est, ce n'est pas l'imaginaire sexuel masculin le plus glorieux. Et j'avais envie de partir de ça comme point de départ d'un autoportrait. Donc j'avais une forme de prudence dans ce travail autour du sexuellement explicite, sans doute aussi parce qu'on est le fruit de constructions culturelles, morales et que ce n'est pas la même chose de travailler autour d'actes de pénétration qu'autour du genoux ou sur le porté. J'avais besoin de gens qui politiquement savaient pourquoi ils venaient, qui étaient très au clair sur leur place dans ce champ et qui avaient une volonté, impulsée par eux, de travailler sur le sexuellement explicite. Cela permettait d'éviter l'enjeu de la manipulation ou de l'intrusion, surtout dans un contexte de travail qui peut être très tendu économique et où les interprètes se retrouvent à accepter des choses parfois difficiles pour eux.

(nou) de Mathieu Hocquemiller, 2014

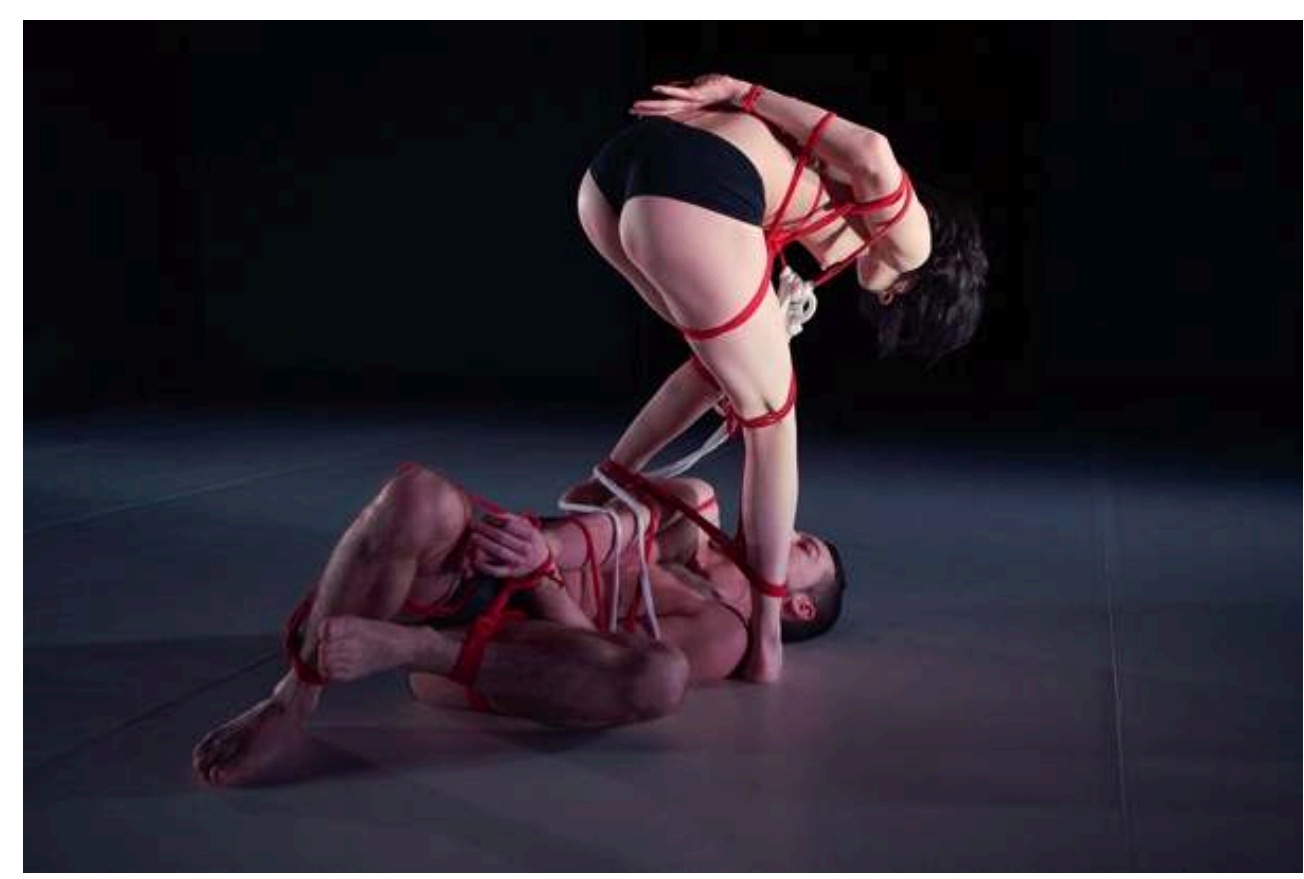

Festival Montpellier danse

\section{Trouver les conditions politiques du consentement}


B. MC. Donc tu cherchais à clarifier les conditions politiques de leur exercice de la représentation?

M. H. Oui. Et il est vrai que dans cet exercice politique-là, le sexuellement explicite pose énormément de questions qu'on devrait d'ailleurs se poser de manière plus large par rapport à l'interprète. Mais comme c'est sexuellement explicite, la question du consentement se pose de manière très forte. Ceci dit, il faudrait définir ce qu'est l'explicite: est-ce que lever un bras pour quelqu'un ça ne met pas en jeu de l'explicite? Ça pose plein de questions intéressantes et demande de redéfinir des catégories... À cet endroit-là, je me suis donc imposé beaucoup de précautions à la fois dans le processus de travail - la question du consentement doit toujours être posée et reposée - et dans la distribution. De ce point de vue, comme Kay vient du porno féministe et queer, il a pu apporter des protocoles de travail de la pornographie féministe où les acteurs procèdent par discussion : chacun énonce ce qu'il peut faire, avec qui, pour rendre un endroit travaillable, ce n'est pas le réalisateur qui dit «Tu fais ça ». Moi, j'arrivais sur (nou) avec des images mentales, mais qui ont rencontré ce protocole de travail et d'échange sur la visibilité. Et je me suis rendu compte que j'avais énormément de précautions et que ce travail-là m'invitait à m'emparer de choses qui pouvaient me mettre à un endroit d'inconfort. Ils voulaient notamment travailler autour de l'urine. Il se trouve que dans ma vie personnelle ce n'est pas forcément mon endroit de fétiche ou de plaisir. Et puis je redoutais le côté un peu provoc Jan Fabre, salle gosse, on fait pipi. Alors que leur endroit de travail était beaucoup plus sincère : comment on réintègre ça dans un enjeu de représentation? Ça a été intéressant de voir comment je pouvais m'en emparer.

B. MC. Quelque part vous avez mis en discussion le travail d'écriture en faisant des allers/ retours?

M. H. Il y a eu des allers/retours permanents sur ce qu'on voulait dire et comment. Même si c'est moi qui ai pris en charge l'écriture. Il y a des choses qu'on n'a pas conservées parce qu'il faut aussi pouvoir reproduire le spectacle: on voulait notamment travailler autour de l'anus pour les deux garçons, puisque c'est un endroit de sacralisation du corps masculin qui ne doit pas être pénétré. Et ils m'ont proposé de travailler sur le fist. Je trouvais ça bien de rendre ça travaillable en désarmant l'effroi qui peut accompagner cette pratique. Mais j'étais plus sceptique sur la reproductibilité de l'acte : si on a dix, quinze ou vingt dates ce n'est juste pas possible. En tout cas cette distribution permettait de construire quelque chose de très clair dans le rapport au consentement.

B. M.C. En particulier pour cette pièce, il me semble que tu as été chercher des interprètes pour travailler contre une logique d'invisibilisation à la fois du sexuel mais pour contrer un imaginaire très normé construit autour du sexuel, à savoir majoritairement cisgenre et hétérosexuel. Est-ce que tu dirais cela comme ça?

M. H. Tous les interprètes sont des gens qui vivent tous leur sexualité de manière non-normative, qui en parlent comme ça et souhaitent la représenter sous cette forme-là. Et cela permettait de travailler sur cette double relégation : le sexuel en soi ne doit pas être représenté, soit pour des raisons très morales et conservatrice ( « c'est pas bien »), soit de manière plus insidieuse parce que l'explicite appauvrirait le rapport au sexuel (c'est un sujet qu'il faudrait traiter par l'ellipse). Cette relégation cantonne la pornographie a un certain vocabulaire effectivement pauvre : comme si l'image explicite ne pouvait pas être complexe, déployer un imaginaire. Pour lutter 
contre cette relégation, on souhaitait aussi créer des représentations alternatives puisque la plupart sont très majoritairement hétéronormatives. Et on voulait aussi sortir le sexuellement explicite de la volonté excitatoire qui le cantonne à la vulgarité sous prétexte que cela ne s'adresserait qu'au corps et à la pulsion. L'hypothèse, c'était d'écrire avec des interprètes à partir de cette hypothèse de Foucault : la sexualité est une culture beaucoup plus qu'une pulsion. Et tous ces interprètes avaient en commun de travailler la sexualité comme pratique et donc comme culture. Et comme n'importe quelle culture, elle s'enrichit d'imaginaires et se construit socialement dans un jeu politique et dans un agencement. La représentation participe de cette construction.

B. Cela donne aussi son importance au geste artistique puisque tu viens enrichir et participer de la construction de cet imaginaire.

M.H. Oui. C'est pour cela que le post-porn est peut-être ce qui pourrait permettre de situer cette démarche-là. Parce qu'il me semble qu'il y a deux temps : ce que tu dis, d'abord, à savoir la réappropriation par les minorités sexuelles et par les femmes du vocabulaire pornographique, par rapport à une érotisation du corps féminin par le regard hétérosexuel masculin. Mais où le vocabulaire - et je le dénigre pas - reste dans une intention pornographique, c'est-à-dire excitatoire. Et il y ensuite a un dépassement de la pornographie qui est en train de se mettre en place. Grossièrement d'ailleurs, pour la danse c'est acquis puisqu'il ne s'agit pas de parler « du » sexe mais de parler « à partir » du sexe. Quelqu'un qui danse sait très bien qu'il n'est pas question que de son coude ou de son biceps, à partir de là il parle d'un agencement au monde. L'événement est encore tel quand il y a un geste sexuel ou une partie génitale qu'on ne verrait que lui.

B. MC. Comme s'il y avait une opacité littérale à laquelle on était condamné dès lors qu'on travaillait sur des zones génitales ou sur des actes sexuels.

M. H. L'idée, c'est précisément de dépasser ça : que ça ne fasse plus événement. C'est un peu déceptif, parce que les gens qui attendent une charge libidinale vont être déçus. À partir du génital et du sexuel, on parle d'autre chose avec, effectivement, dans (nou) et Auto-Porn Box des corps non-normatifs. 


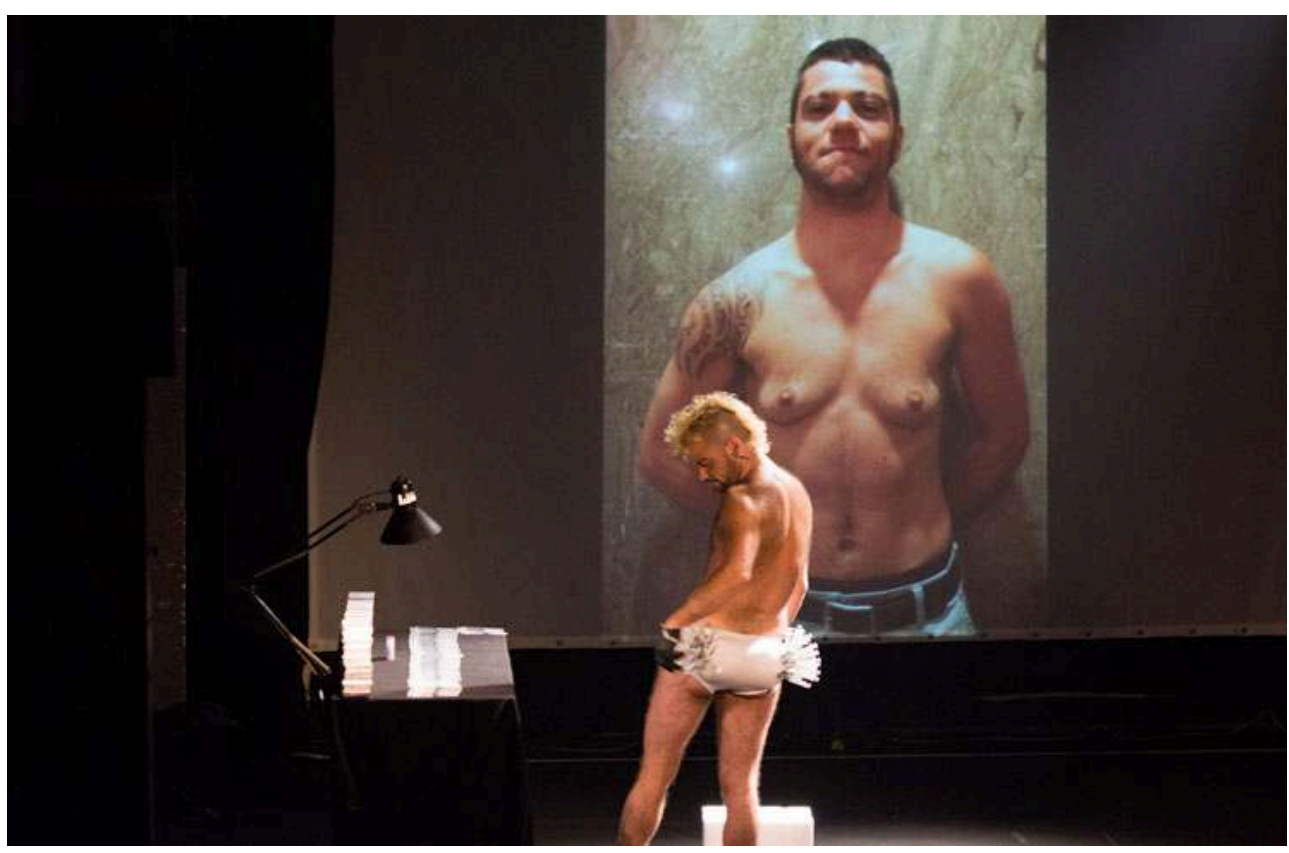

hTh

\section{Le hors-champ de la danse : le circuit militant des travailleur-se-s du sexe, des féministes pro-sexe et des performeur-se-s queer}

B. MC. Comment trouves-tu ces interprètes hors des circuits classiques de distribution des professionnels de la danse? Comment fais-tu pour provoquer ces rencontres?

M. H. Kay, je l'ai rencontré au Porn festival à Berlin où il faisait un workshop sur la transition de genre et j'avais bien aimé son travail. J'ai découvert qu'il faisait du porno queer et qu'il avait notamment travaillé avec Bruce Labruce. Marianne, c'est par le biais d'un article qu'elle avait écrit, publié par le STRASS (Syndicat des Travailleur-se-s du Sexe), et que j'avais trouvé très intéressant. Elle avait écrit un article sur la performativité qui rapprochait le corps du travailleur sexuel de celui de l'interprète au plateau. Par ailleurs, je l'avais vue sur scène sans savoir que c'était elle. Mathieu, je l'avais rencontré à un Gender Lab organisé par Beatriz [Paul] Préciado et il avait comme moi ce double profil militant queer et artiste avec une forte technicité puisqu'il est aussi chanteur lyrique.

B. MC. Donc tu as été chercher des gens qui étaient aussi à un endroit de théorisation de leur pratique.

M. H. Oui, c'est ce que j'appelais la « conscience politique » car je voulais travailler avec des gens lucides, au clair sur ce questionnement et sur ce qu'ils sont prêts à faire et pourquoi. Je voulais éviter toute forme de projection. Donc le système de rencontre de ces interprètes était essentiellement celui du réseau militant et queer. Je crois que ce qui m'intéresse c'est de travailler à questionner la norme des corps représentés et des imaginaires. L'intérêt est artistique - dans l'histoire de la représentation - et politique dans l'enrichissement des possibilités. Je trouve assez joli ce que dit Butler : 
«Qu'est-ce qui rend des vies vivables?» Question qui nous invite à interroger les modalités de l'inclusion.

B. MC. Est-ce que tu te sens concerné dans tes processus de distribution par l'invisibilisation des interprètes racisés ou par leur distribution dans un cadre souvent très stéréotypé ?

M. H. Avec Jusque là c'est nous, j'ai travaillé avec Hurlande qui est noire mais ce qui m'intéressait c'était son parcours de migration plus que le critère spécifiquement racial. Je crois que ce qui fait que je n'aborde pas frontalement cet enjeu c'est parce que je m'en sens moins proche. Par exemple dans les Auto-Porn Box, je peux aborder la question de la transition de genre à travers l'autoportrait de Kay, parce qu'il est directement concerné et qu'il a décidé de travailler là-dessus. Je n'aurais pas abordé la question trans moi-même. Je me sentirais mal à l'aise. Je ne dis pas qu'il n'y a que les acteurs concernés qui soient légitimes pour en parler. Mais je trouve ça compliqué. C'est la question que pose Exhibit $B$ de Brett Bailey : s'il avait mis en scène le corps dominant, le colon blanc, ça aurait pu être différent. C'est peut-être une esquive mais j'ai l'impression que plus ça va et plus j'essaye de travailler avec l'endroit d'où l'on parle. Par exemple, on réfléchit à un duo avec Mathieu [Jedrazac] sur le corps masculin dans une perspective féministe, en prenant au pieds de la lettre ce que Virginie Despentes dit dans King Kong théorie: "Nous les meufs on a fait le boulot, on s'est réapproprié notre corps, mais le corps masculin n'est pas interrogé alors allez-y ». J'aimerais bien partir d'angles morts de la représentation du corps masculin.

B. MC. Est-ce que tes principes de distribution ont changé au fur et à mesure de tes créations ou est-ce que tu as reconduit ce principe de rencontres provoquées?

M.H. J'ai l'impression que j'ai toujours travaillé à partir de rencontres mais que les thématiques que je traitais ont eu un effet de déplacement puisque, comme je te le disais, je ne souhaitais pas travailler exclusivement avec des danseurs. En ce moment, j'aimerais reprendre le plateau en partageant l'endroit de la décision artistique sous la forme de deux duos, l'un avec Marianne et l'autre avec Mathieu.

B. MC. Donc tu vas te redistribuer toi-même?

M. H. Oui! (Rires) Tout ça pour ça!

\section{Interroger les processus de distribution de la culture}

B. MC. Est-ce qu'en travaillant sur ce type de sujet, et en organisant le festival Explicit, consacré aux formes artistiques qui travaillent sur/à partir du sexuel à hTh qui est une institution, tu as le sentiment de travailler sur la manière dont se distribuent les formes culturelles? Est-ce que tu interroges les processus de distribution de la culture elle-même par rapport à ses propres territoires de légitimité ou d'illégitimité ?

M. H. Oui. En tout cas, j'ai clairement l'envie d'offrir des espaces où ces formes-là soient visibles. Comme le discours performatif produit quelque chose, je crois qu'en ouvrant ces espaces-là, consacrés au post-porn, dans un lieu comme une scène nationale, cela participe à un déplacement des lignes. Je crois que dans le festival Explicit, il y avait une double adresse. Une adresse au milieu politique, underground, DIY pour rappeler l'exigence artistique et le travail sur la forme : qu'est-ce qu'on raconte à partir du sexuel ? Et une adresse à l'univers artistique pour dire : on peut travailler là-dessus sans être condamné à l'ellipse. 
B. M.C Et par exemple, une pièce comme (nou), quel est son réseau de distribution?

M. H. C'est une pièce qui a aussi été très malmenée par le contexte de sa création puisque avec les grèves de l'année dernière il y a eu pas mal de problème. Mais c'est vrai que c'est une pièce qui a pu permettre une forme de convergence puisqu'on a joué dans des lieux underground et d'autres plus institutionnels.

B. MC. Est-ce que tu crois que ça permet aussi d'interroger les dispositifs qui font qu'une culture est considérée comme plus ou moins légitime, non?

M. H. Oui. En tout cas c'est ce qu'on aimerait. Et cette exigence elle était là aussi dans Explicit. Pour la programmation, on a pu avoir du mal par exemple a trouver des formes artistiques de qualité - extraire des pépites comme les films d'Antonio Da Silva, c'est donc un geste performatif. C'est rendre des choses possibles pour qu'elles existent. 